\title{
La Moneda Social y Complementaria como herramienta de intercambio en el aula de Economía
}

\section{David Blanco-Alcántara ${ }^{a}$ y Borja García-Villafranca ${ }^{b}$}

aProfesor del Departamento de Economía y Administración de Empresas de la Universidad de Burgos. Email: dblanco@ubu.es, ${ }^{b}$ Coautor de contacto. Profesor Asociado del Departamento de Economía y Administración de Empresas de la Universidad de Burgos. Profesor de Educación Secundaria en la especialidad de Economía. Email: borjagarciav@gmail.com

\begin{abstract}
In this study we describe a project that implements an exchange tool, specifically a Social and Complementary Currency, in teaching area. The aim, with a series of questions before and after the currency uses, is to asses whether they have met a series of objectives that we have already set. And it will help us to know if it has potential as a teaching tool. These objectives cover different areas, some of them are social (to improve the relationship between students), other academic ( increase financial literacy and motivation) and others to promote individual initiative and decision making. We will check, taking into account a number of limitations, how this tool will achieve most of the proposed objectives and we will get a guide for future researches.

Keywords: social and complementary currency, exchange, market, tool, motivation, economy, educational innovation.
\end{abstract}

\footnotetext{
Resumen

En esta investigación se describe un proyecto en el que se pone en práctica una herramienta de intercambio en el entorno educativo, en concreto una Moneda Social y Complementaria. La finalidad, con una serie de preguntas previas y otras posteriores al uso de la moneda, es poder evaluar si se han cumplido una serie de objetivos que se han marcado y que servirán para conocer su posible potencial como herramienta pedagógica. Estos objetivos cubren diferentes ámbitos, unos de corte social (como mejorar la relación entre alumnos), otros a nivel académico (como aumentar la cultura financiera y la motivación) y otros de fomento de la iniciativa individual y toma de decisiones. Se comprueba como tras su utilización, y teniendo en cuenta una serie de limitaciones, esta herramienta va a conseguir potenciar la mayoría de los objetivos propuestos y servirá de guía para futuras investigaciones.
}

Palabras clave: Moneda Social y Complementaria (MSC), intercambio, mercado, herramienta, motivación, economía, innovación educativa. 


\section{Introducción}

Este trabajo versa sobre la investigación de la capacidad pedagógica de una Moneda Social y Complementaria (en adelante MSC) en el aula. La idea es conocer las virtudes que puede poseer una MSC como herramienta pedagógica en un entorno educativo. Y poder, de esta manera, saber más en profundidad su posible potencial en el sector educativo.

En la enseñanza de economía se observan dificultades por parte del alumno en la construcción del mundo económico. Estas dificultades son debido a su compleja estructura sistemática y a su relación con el sistema social. Así, se antoja imprescindible acercar la cultura financiera a todas las personas, pues son ellas quienes hacen economía cada día y, en concreto, a nuestros alumnos. Es por ello, que se considera que esta innovación docente contribuye a afrontar ciertos contenidos del mundo económico, como es el sistema monetario y múltiples conceptos que giran sobre el mismo, de una manera más lúdica y participativa, siendo el alumno eje central de la misma.

Una de las principales causas del fracaso escolar es la falta de atención en clase, poca motivación y apatía del alumnado al propio proceso de formación. Una solución para ello: la participación del alumnado en el propio proceso de aprendizaje, "Aprender haciendo". Y trabajar en los cuatro componentes que según Martin Gordillo (2006) son necesarios para una educación integral: educar para conocer, educar para manejar, educar para valorar y educar para participar.

Antes de exponer los objetivos que se persiguen con esta innovación docente se dedicará unas líneas a introducir el concepto de MSC y concretar qué tipo de moneda es el escogido para este proyecto.

"Moneda social", fue una expresión inventada por Heloísa Primavera, tras observar las experiencias de los clubes de trueque que emergían en aquel entonces en Argentina. El fin era destacar "sus efectos de inclusión social" (Primavera, 1999) a través de la "creación de una nueva masa monetaria de circulación restringida a los participantes de los clubes".

Existen diferentes tipos de MSC, cada una con sus propias características y objetivos, pero en general todas ellas tienen unos objetivos comunes (Blanc, 2009): localización, dinamización y transformación de la naturaleza de los intercambios. Aclarar que no se procede a exponer en este trabajo los tipos de MSC que existen por no ser un trabajo de investigación sobre MSC y por la gran extensión que conllevaría. Lo que sí se define es el tipo de MSC que se implementará en el aula, que es el sistema LETS. Se ha elegido este sistema por su fácil implementación y adecuación al entorno del aula. A continuación, se explica sucintamente este tipo de herramienta de intercambio que se creará y se pondrá en funcionamiento en el aula.

\section{Sistema LETS:}

El sistema más conocido de Monedas Sociales y Complementarias (MSC) a nivel mundial es LETS que nació en Commox Valley, Columbia Británica, Canadá en 1982. Después se ha expandido a otros países, sobre todo en Reino Unido, Australia, Francia (donde se denomina "SEL" (Systèmes d'Échanges Locaux)) y Alemania (donde se denomina 
"Tauschring"). Con este sistema no circulan billetes ni monedas sino que cada socio (o participante de la moneda) crea su cuenta donde se registra su saldo (Hirota, Y., 2013). Por ejemplo, si Elena compra a Fernando un libro que vale $10 €$, se registra esta transacción en el ordenador de la oficina de este LETS y el saldo de Elena se queda con 10 puntos negativos mientras que el de Fernando está con 10 positivos.

Normalmente estos grupos no son grandes y muy pocos sistemas han conseguido reunir más de 200 socios activos debido a los controles débiles (Douthwaite, 1999).

Este sistema tiene las siguientes características (Hirota, Y., 2013):

- La suma de todas las cuentas es siempre de cero. Alguien tiene que quedarse con saldo negativo para que otro(s) esté(n) con el positivo.

- Las cuentas permiten llegar hasta un saldo negativo de -X (ejemplo -100) definido previamente. Por lo que puedes empezar a utilizar la moneda al momento. Inicialmente las cuentas están a 0 .

- No se cobra ninguna tasa de interés a los saldos negativos.

Un ejemplo de una MSC tipo LETS en España es: "La moneda Puma" que está dinamizando un barrio de la ciudad de Sevilla.

A continuación se exponen distintos factores importantes para una exitosa puesta en marcha de LETS (North, 2010). Si bien, hay que tener en cuenta que estos factores han sido analizados para poner en marcha un LETS en un entorno ajeno al ámbito educativo:

1. Evitar dos errores fundamentales: el de generar expectativas excesivas (solución: no exagerar los efectos de esta herramienta) y el de aferrarse al diseño inicial del sistema (solución: ser flexible y adaptarse a los contextos socioeconómicos locales).

2. Introducir la gente al sistema de forma adecuada: explicación a los nuevos socios cómo funciona el sistema y confirmación de su comprensión perfecta.

3. Gestión profesional del sistema: elaboración de materiales publicitarios y directorios bien diseñados y organización de fiestas y viajes, entre otros, para que se conozcan los socios.

4. Suficiente variedad de capacidades ofrecidas para cubrir las necesidades de la gente: garantizar una mínima oferta, tanto de bienes como de servicios, que permita los intercambios.

5. Espiritu bien definido y compartido: construcción de un consenso sobre el mecanismo y el objetivo del sistema.

6. Avisar a cada socio lo que se consigue de LETS: transacción de bienes y servicios no profesionales que no se venderían en dinero de curso legal.

7. Cuidar a los socios activos: intervención para que los socios con talentos, capacidades, etc., que todo el mundo quiere, no acaben acumulando demasiado saldo positivo, se cansen

(cc) EY-NC-ND 2016, Universitat Politècnica de València

Congreso In-Red (2016) 
de ofrecer este servicio y abandonen la MSC (limitación del máximo saldo positivo e intermediación de servicios, etc., para que gasten estos socios activos).

8. Aclarar bien los aspectos relacionados con los beneficios fiscales: la oferta de servicios no profesionales está exenta de fiscalización. No se está realizando nada ilegal, o que vaya a ser prohibido por alguna Administración.

Si bien, como se mencionó anteriormente, estas pautas no son específicas para un entorno educativo, pero sirven como referencia para una correcta implantación de la MSC en el aula, analizando posibles divergencias con su aplicación en otros ámbitos.

La puesta en marcha de una MSC, conlleva la creación de un mercado, aunque no es necesario que exista físicamente, pues hay mercado cuando se produce un intercambio.

\section{Objetivos}

Como se comentó en el punto anterior, el objeto de la investigación es analizar la capacidad pedagógica de una MSC tipo LETS en el aula. Si bien, este objetivo tan amplio debe ser matizado para poder contrastar su consecución. Así, se establecen objetivos en diferentes ámbitos: unos de corte social (como mejorar la relación entre alumnos), otros a nivel académico (como aumentar la cultura financiera y la motivación) y otros de fomento de la iniciativa individual y toma de decisiones.

De esta manera, se concreta explícitamente los seis objetivos que se persiguen, y que serán evaluados, en esta innovación docente:

a) Motivar a los alumnos en su aprendizaje.

b) Mejorar el conocimiento de los contenidos relacionados con Monedas y Mercado.

c) Estimular el "saber hacer" y la capacidad emprendedora.

d) Asumir estrategias de actuación, actividad y reflexión económica.

e) Generar intercambios de bienes y servicios en el aula.

f) Mejorar las relaciones entre los alumnos.

A continuación, se procede a explicar en unas breves líneas cada uno de ellos.

a) Motivar a los alumnos en su aprendizaje. La desmotivación del alumno es uno de las causas principales de fracaso escolar. La idea es llevar al aula una herramienta que active la curiosidad e interés del alumno. Algo nuevo, que no hayan tratado antes y que suscite su atención y reflexión.

b) Mejorar el conocimiento de los contenidos relacionados con Monedas y Mercado. La MSC tipo LETS tiene las características de una herramienta de intercambio. En este caso será una herramienta creada por ellos y para ellos. Esto debería dar una nueva dimensión a su manera de entender estos conceptos. Además con su desarrollo, se creará un 
mercado, en el cual entran en juego factores como oferta, demanda, precios y todo lo que con su desarrollo está relacionado: competencia, calidad de producto o servicio, políticas monetarias expansivas y restrictivas, inflación...

c) Estimular el "saber hacer" y la capacidad emprendedora. Al crear un mercado que necesita de bienes y servicios, y donde los alumnos pueden ver qué demandan los demás compañeros, existe un caldo de cultivo donde el alumno puede reflexionar lo que puede ofrecer, lo que sabe hacer y lo que es más demandado. Con ello se podrá analizar si se estimula el saber y querer hacer. Por otra parte, es una herramienta creada por los alumnos y que gestionan ellos mismos, estimulando por tanto la autonomía del individuo, la toma de decisiones y la capacidad emprendedora.

d) Asumir estrategias de actuación, actividad y reflexión económica. Tener una herramienta de intercambio en el aula en forma de MSC podría dar lugar a reflexiones económicas por parte de los alumnos. Y, al profesor, a utilizarlo como ejemplo para impartir los diferentes contenidos de la materia. Recuerdese como se antoja necesario ahondar en la cultura financiera de las personas.

e) Generar intercambios de bienes y servicios en el aula. Este, en principio, es el motivo principal de una herramienta de intercambio. Por lo tanto, se analizará el tipo y cantidad de intercambios que se realizan en el aula. Si bien, en este caso concreto, los objetivos pedagógicos serán prioritarios.

f) Mejorar las relaciones entre los alumnos. El poder compartir servicios con los compañeros, podrá dar lugar a que se relacionen más entre ellos y que se conozcan mejor.

Para la contrastación y análisis de la consecución de estos objetivos se realizará una encuesta previa a la innovación docente y otra posterior, donde parte de la información se solape y nos permita comparar lo sucedido. La explicación de la metodología empleada y el desarrollo de la innovación docente deriva al siguiente apartado del presente trabajo.

\section{Desarrollo de la innovación}

En el desarrollo de la innovación se puede diferenciar una serie de pasos, donde la puesta en práctica y el acierto de la herramienta de intercambio pueden influir en el éxito o fracaso de la investigación. Como ya se mencionó al comienzo, el tipo de MSC escogida es del tipo LETS, y en el apartado anterior se expuso la realización de una serie de preguntas, encuestas, que permitirán valorar la consecución de los objetivos.

Antes de exponer dichos pasos se explicará el marco en el que se ha desarrollado dicha innovación docente, es decir, lo que se puede considerar la muestra de estudio.

Para la aplicación de esta innovación docente se contó con la colaboración de un instituto de la localidad de Sevilla. Dicho centro es de tituralidad pública, y se encuentra situado en un barrio de los más populares e importantes de Sevilla, su ubicación geográfica coincide prácticamente con el centro de la ciudad.

(cc) EY-NC-ND 2016, Universitat Politècnica de València

Congreso In-Red (2016) 
Desde el año 2010, con los resultados de las pruebas de diagnóstico, se facilita al Centro un indicador del nivel socioeconómico y cultural de las familias (ISC), a partir de cuestionarios que rellenan ellas mismas. El instituto tiene una puntuación que, en el conjunto de Andalucía, representa un valor medio.

Se escogieron dos grupos-clases diferentes, ambos pertenecientes a $1^{\circ}$ bachillerato de la modalidad de ciencias sociales y que cursaban la asignatura de Economía. El primer grupoclase estaba constituido por 22 alumnos y el segundo grupo-clase por 9 alumnos. Así, cada grupo-clase trabajó de forma independiente la MSC, creando cada uno su propia moneda. La innovación docente se desarrolló en el curso 2013-14 durante 1 mes, concretamente en los meses de marzo-abril. Si bien, se debe especificar que la temporalización se distribuyó de la siguiente manera: una primera semana orientada a conocer el funcionamiento de una MSC; las tres siguientes semanas ya fueron de implementación y funcionamiento de la MSC; y, finalmente, una última sesión (clase) para la recogida de información y evaluación de la experiencia. Hay que señalar que el tiempo dedicado a la innovación se considera escaso, como se recoge en las valoraciones realizadas por los propios alumnos, siendo este un factor a mejorar para sucesivas experiencias. Pero lo que se perseguía con este proyecto era analizar una primera experiencia y viabilidad de la innovación antes de desarrollar un proyecto más completo e incluso su implementación en la enseñanza universitaria. Además, sirve como un punto de unión entre el ámbito universitario y enseñanzas medias, aspecto que se considera de gran importancia.

Una vez concretado el escenario donde se articula la innovación docente, se expondrán los pasos de su desarrollo, siendo cinco:

a) Preguntas previas.

b) Explicación de las herramientas de intercambio.

c) Creación de una herramienta de intercambio.

d) Desarrollo de la herramienta de intercambio, periodo en el que está funcionando la MSC.

e) Análisis y valoración.

El primer paso es la recogida de datos iniciales, que nos permitirán contrastar una vez desarrollada la innovación los resultados y por tanto valorar la consecución de objetivos. Así, se hace uso de una encuesta que incluye principalmente preguntas de tipo escala Likert y alguna pregunta abierta, combinando por tanto metodología cuantitativa y cualitativa. Se debe señalar que la escala Likert es una escala psicométrica comúnmente utilizada en cuestionarios y es la escala de uso más amplio en encuestas para la investigación, principalmente en ciencias sociales. Al responder a una pregunta de un cuestionario elaborado con la técnica de Likert, se especifica el nivel de acuerdo o desacuerdo con una declaración. La escala en las respuestas en este caso serán del 1 al 5, siendo el 1 "totalmente en desacuerdo", 2 "en desacuerdo", 3 "ni de acuerdo ni en desacuerdo", 4 "de acuerdo" y el 5 "totalmente de acuerdo".

(c)) BY-NC-ND 2016, Universitat Politècnica de València 
Seguidamente a la recogida de datos se procede a explicar qué es una MSC, cómo funciona, etc... Es decir, la parte conceptual que se hablaba en la primera fase de la temporalización.

Una vez expuestos los conocimientos más básicos se procede al tercer paso, la creación de la MSC. Se recuerda como cada grupo-clase creaba su propia MSC, siendo tipo LETS. Ambas presentan características idénticas, permitiéndose saldos negativos pero con un límite de -50 .

Así, el cuarto paso es la puesta en escena de la MSC. Durante 3 semanas como se expuso en la temporalización los alumnos pudieron estar operando con su MSC.

Por último, el quinto paso, desarrollado en una última sesión, consistió en la recogida de nuevo de datos por medio de otra encuesta. En este caso, se incluían preguntas tipo Likert, algunas de ellas idénticas a las recogidas en la encuesta inicial para favorecer una comparación directa y poder obtener conclusiones más exactas, y otras preguntas abiertas. Así mismo, se realizó una evaluación a los alumnos sobre la experiencia de la MSC y de la labor desempeñada por el profesor.

De forma que, como se puede apreciar, se realiza una recogida de datos previa a la aplicación de la MSC y otra posterior. Con estos datos se procede a comparar las respuestas obtenidas, lo que permitirá analizar la consecución de los objetivos planteados. Este análisis, por motivos de extensión, se realiza en el apartado de resultados, donde.

En el apartado de resultados también se mostrará un análisis DAFO, realizado sobre la MSC, como parte de ese último paso de análisis y valoración.

\section{Resultados}

Como se mencionó en el anterior apartado, a continuación se exponen las tablas que muestran conjuntamente las preguntas, respuestas y, en su caso, diferencias entre el antes y después. La tabla 1 recoge las preguntas Likert, diferenciando si la pregunta se realizó antes, antes y después o después de la experiencia de la MSC. Así se puede observar que en el caso de aquellas preguntas que se realizaron tanto antes como después se incluye la diferencia entre ambos valores (columna DIF), permitiéndose una comparación directa y facilitando el análisis y valoración de la consecución de los objetivos. Así mismo, se incluye una última columna que muestra el objetivo/s con los que guarda relación más directa la pregunta y, que por tanto, las respuestas a la misma ayudarán a analizar la consecución del mismo.

En la tabla 2 se recogen las preguntas de tipo abiertas y las respuestas a las mismas. Como se puede observar la primera de ellas es la que se corresponde al cuestionario inicial (previo al desarrollo de la innovación docente) y las siguientes 5 preguntas son posteriores. En este caso las respuestas se categorizan, si procede según el tipo de pregunta, en valoración positiva o valoración negativa. $\mathrm{E}$, igualmente, se muestra una última columna (OBJ.) que recoge el objetivo con el que la pregunta guarda mayor relación, sirviendo por tanto para analizar el grado de consecución del mismo.

(cc) EY-NC-ND 2016, Universitat Politècnica de València

Congreso In-Red (2016) 
Tabla 1. Preguntas tipo Likert y sus respuestas.

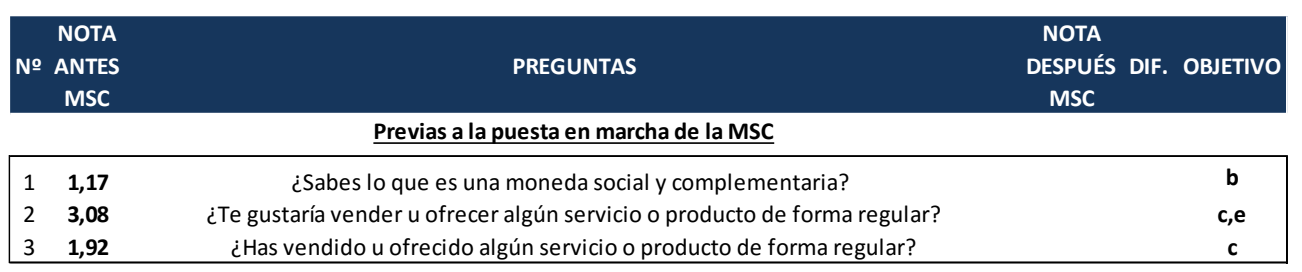

Antes y después de la puesta en funcionamiento de la MSC

\begin{tabular}{|rrrrrrr|}
\hline 4 & $\mathbf{3 , 1 7}$ & ¿Conoces los requisitos y funciones que tiene que tener una moneda? & $\mathbf{3 , 7 1}$ & 0,54 & b \\
5 & $\mathbf{3 , 1 7}$ & ¿Crees que te será útil usar una herramienta de intercambio creada por y para vosotros? & $\mathbf{3 , 2 9}$ & 0,12 & a,d \\
6 & $\mathbf{3 , 5 8}$ & ¿Piensas que tus compañeros podrían ofrecerte productos o servicios útiles para ti? & $\mathbf{3 , 8 7}$ & 0,29 & $\mathbf{d}$ \\
7 & $\mathbf{3 , 0 3}$ & ¿Podrías ofrecer algún bien o servicio en la escuela? & $\mathbf{3 , 3 7}$ & 0,34 & $\mathbf{c , e}$ \\
8 & $\mathbf{3 , 3 8}$ & ¿Podrías ofrecer algún bien o servicio fuera de la escuela? & $\mathbf{3 , 3 9}$ & 0,01 & $\mathbf{c}$ \\
\hline
\end{tabular}

Posteriores a la puesta en marcha de la MSC

\begin{tabular}{|crrr|}
\hline 9 & ¿Conoces los requisitos para constituir (conformar) una moneda? & $\mathbf{3 , 7 1}$ & b \\
10 & ¿Crees que han aumentado tus conocimientos sobre qué es una moneda? & $\mathbf{3 , 9 6}$ & $\mathbf{b}$ \\
11 & ¿Te gustaría conocer más sobre monedas sociales y complementarias? & $\mathbf{3 , 1 4}$ & $\mathbf{a}$ \\
12 & ¿Os ha gustado la experiencia? & $\mathbf{4 , 0 9}$ & $\mathbf{a}$ \\
13 & ¿Os ha ayudado a conoceros más entre vosotros? & $\mathbf{2 , 9 8}$ & $\mathbf{f}$ \\
14 & ¿Has recibido bienes o servicios del grupo que te han sido útiles? & $\mathbf{2 , 6 1}$ & $\mathbf{e}$ \\
15 & ¿Cuántos bienes o servicios has ofrecido? & $\mathbf{0 , 6 5}$ & $\mathbf{e}$ \\
16 & ¿Te gustaría empezar con la moneda al principio de clase y mantenerla todo el curso? & $\mathbf{3 , 7 9}$ & a,e \\
\hline
\end{tabular}

Fuente: Elaboración propia.

Con el objetivo de facilitar la comparación entre el antes y después de aquellas preguntas iguales entre ambos momentos temporales se recogen las diferencias entre las respuestas de las mismas en el gráfico 1:

Gráfico 1: Comparativa de respuestas antes y después de la innovación.

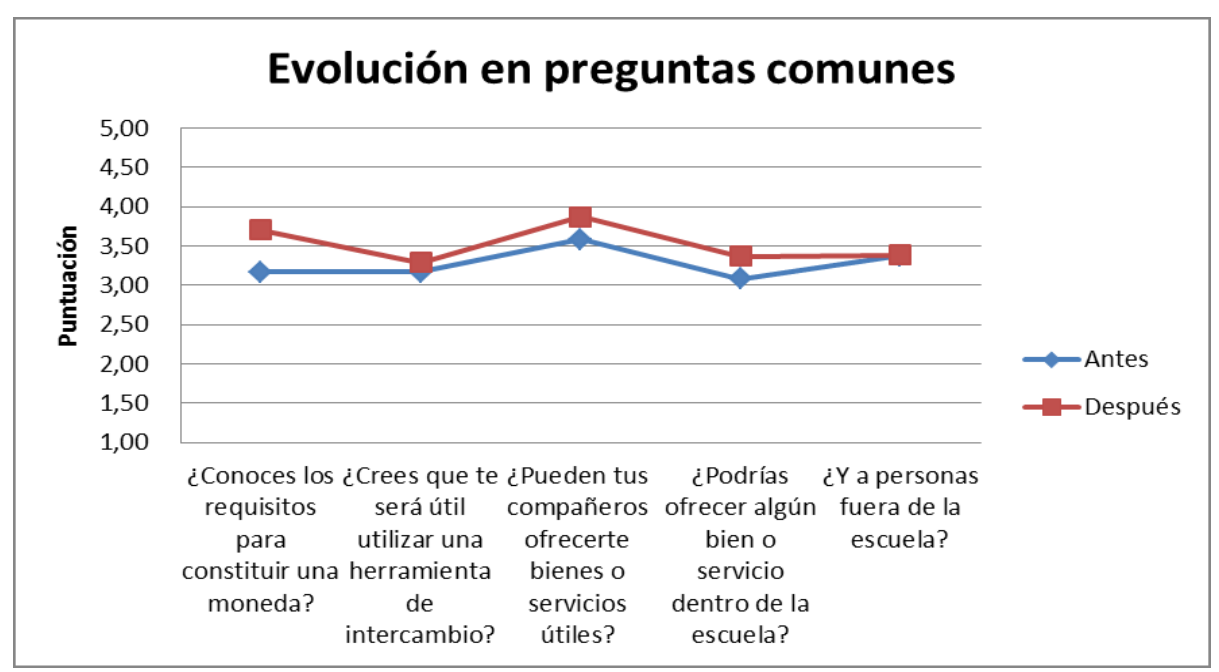

Fuente: Elaboración Propia.

(cc) EY-NC-ND 2016, Universitat Politècnica de València

Congreso IN-RED (2016) 
Tabla 2: Preguntas de tipo abierta y sus respuestas

Previas a la puesta en marcha de la MSC

\begin{tabular}{|c|c|c|}
\hline 1 & $\begin{array}{c}\text { ¿Por qué crees que te será útil utilizar una herramienta de intercambio creada por y para } \\
\text { vosotros? }\end{array}$ & \\
\hline \multirow{2}{*}{$\begin{array}{l}\text { Valoración } \\
\text { positiva }\end{array}$} & $\begin{array}{l}\text { "Porque las herramientas que utilizaremos serán de beneficio para los demás compañeros", } \\
\text { "Sabríamos que es lo que necesitamos", "Sería acorde a nuestras necesidades", "Se evitarían } \\
\text { conflictos y nos ayudaríamos más" }\end{array}$ & f \\
\hline & $\begin{array}{l}\text { "Porque nos facilitaría la comprensión en la economía", "Sería una manera interesante para } \\
\text { aprender más acerca del dinero y las monedas" }\end{array}$ & \\
\hline Valoración & $\begin{array}{l}\text { "Poco, porque no tendría valor fuera de aquí," "No tendría valor real económico", "No tiene } \\
\text { apoyo de los demás países" }\end{array}$ & d \\
\hline & "Creo que no la utilizaremos", "será un lío". & \\
\hline
\end{tabular}

Posteriores a la puesta en marcha de la MSC

2 ¿Por qué crees que te será útil utilizar una herramienta de intercambio creada por y para vosotros?

"Para adquirir bienes y servicios sin pagar euros","Facilita a tener más recursos", "Se puede adaptar a nuestras necesidades"

Valoración "Porque me ayuda a cambio de algo o de alguna ayuda que pueda ser útil a mis compañeros",

positiva "Nos sirve para divertirnos y relacionarnos más entre nosotros", "Porque serviría de ayuda para mejorar las relaciones en clase"

"Nos ayuda a comprender conceptos y como utilizarlos"

Valoración "No tenemos tantos medios de intercambios, y creo que no se llegará a realizar los negativa intercambios", "No lo veo útil"
"Porque he aprendido mucho además de una manera diferente y más divertida", "Actividad creativa", "Las actividades en grupo me gustan"
Valoración "Ha ayudado a conocer q necesitan mis compañeros y así acercar nuestras relaciones", "Ser positiva mejores compañeros"
"Porque hemos realizado intercambios útiles", "Nos enseña a como tener más recursos"
Valoración "Creo que sí tuviéramos más tiempo sería mucho mejor", "Ahora vienen los exámenes finales y negativa muchos compañeros ofrecen clases de apoyo que nos vendrían bien a todos/as"

\section{4 ¿Qué es lo que más te ha gustado de la moneda?}

"Poder ayudar a mis compañeros", "Poder ver los bienes y servicios que ofrecían mis

compañeros", "Mejora las relaciones sociales", "Que beneficia a la sociedad", "Ha ayudado a conocernos más"

Valoración "Su utilidad", "El funcionamiento y desarrollo"

positiva "Lo que más obtener recursos útiles", "El hecho de poder conseguir cosas a cambio de otras, sin tener que gastar dinero", "Que no se necesita dinero real como el euro, ni es controlado por el banco de España"

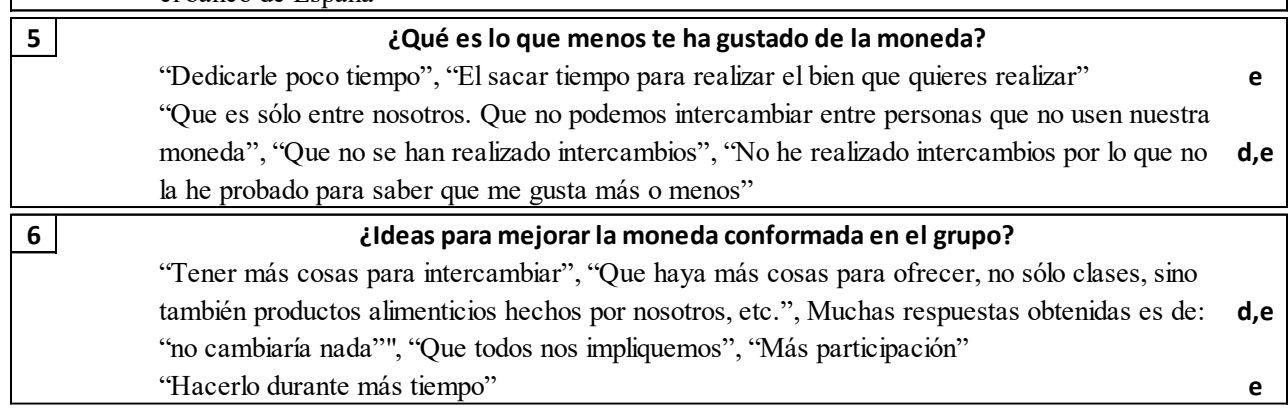

Fuente: Elaboracion propia.

(cc) EY-NC-ND 2016, Universitat Politècnica de València

Congreso In-Red (2016) 
En relación a las respuestas obtenidas se puede decir que, antes de introducir la MSC, los alumnos a la pregunta de si pensaban que la herramienta iba a ser útil o no, un buen número de los comentarios con valoración negativa eran relativos a que la moneda no tendría valor real, porque no era oficial ni era aceptada en otros países. La idea de los alumnos era que las monedas deben ser oficiales y extensamente utilizadas y aceptadas para que estas tengan utilidad. De lo contrario no tienen ningún valor de uso. Piensan a nivel macro, y no a un nivel micro, a nivel local; por el contrario, después de haberla utilizado cambia su argumento a "que haya más ofertas que cubran sus necesidades". Aquí se ha dado un cambio de percepción desde nivel macro a micro. Ya no es importante que estén respaldadas o aceptadas por una institución oficial, sino que les sea de utilidad. Se pasa de lo global a lo local.

En un gran número de las preguntas de opinión aparece como respuesta que el tiempo empleado para su desarrollo ha sido insuficiente, que les gustaría tener más. Efectivamente, tal y como ya se expuso en líneas anteriores, ésta es una de las limitaciones presentes en esta innovación docente y que se debe mejorar en sucesivas ocasiones. La herramienta (la MSC) estuvo en funcionamiento tres semanas desde que se puso en marcha, considerándose tiempo insuficiente, pero se recuerda como se pretendía analizar una primera prueba piloto que sirviera para cotejar la viabilidad de dicha innovación. Añadir que este motivo y el que el investigador no pudiera estar en clase supervisando el desarrollo de la herramienta, fueron la razón principal por la cual el número de intercambios haya sido muy reducido, 0,65 por persona.

A continuación, se analizará lo acontecido con cada uno de los seis objetivos propuestos en esta innovación docente $\mathrm{y}$, a corde a las respuestas obtenidas tanto en las preguntas Likert (tabla 1) como en las preguntas abiertas (tabla 2), se obtiene que:

Se ha logrado el primer objetivo, "Motivar a los alumnos en su aprendizaje". Las razones que conducen a esta afirmación son, entre otras, las respuestas obtenidas en las siguientes preguntas:

- $\quad 3,14$ pts. sobre 5 en la pregunta $n^{\circ} 11$ : “ite gustaría conocer más sobre monedas sociales y complementarias?”.

- 4,09 pts. en la pregunta $n^{\circ} 12$ : “ $i o s$ ha gustado la experiencia?”.

- $\quad 3,79$ pts. en la pregunta $n^{\circ} 16$ : “¿Te gustaría empezar con la moneda al principio de clase y mantenerla todo el curso?".

- En respuestas de opinión. Cuando se les ha hecho la pregunta no 3: “¿Os ha gustado la experiencia? ¿Por qué?" la respuesta más contestada ha sido: "Porque he aprendido mucho además de una manera diferente y más divertida", "Ha sido una actividad creativa".

El segundo objetivo "Mejorar el conocimiento de los contenidos de la unidad de Monedas y mercado" se considera que se ha alcanzado tras valorar las siguientes respuestas:

(cc)) EY-NC-ND 2016, Universitat Politècnica de València 
- En la pregunta $n^{\circ} 4$ realizada antes y después del uso de la moneda social “¿Conoces los requisitos y funciones que tiene que tener una moneda?" se ha obtenido un 3,71. Se ha logrado, entre el antes y después, un aumento de $+0,54$.

- $\quad$ En la pregunta $n^{\circ} 10$ : “¿Crees que han aumentado tus conocimientos sobre qué es una moneda?", la nota obtenida es alta, 3,96, evidenciando una mejora de los conocimientos que recoje el segundo objetivo planteado.

- También, en las respuestas dadas de opinión, se aprecia como los alumnos han aprendido sobre el tema. En la pregunta $n^{\circ} 3$ : “ ¿Os ha gustado la experiencia? ¿Por qué?" se encuentran respuestas del tipo "Porque he aprendido mucho además de una manera diferente y más divertida", incluso esta respuesta refuerza la consecución del primer objetivo mostrado anteriormente sobre la motivación.

Sobre el tercer objetivo marcado de "Estimular el saber hacer y la capacidad emprendedora" se observa que los alumnos han reflexionado que hace falta una mayor diversidad de productos y servicios. La mayoría de las ofertas fueron clases particulares sobre las asignaturas que estaban trabajando en el curso escolar. En esta línea se debe señalar la respuesta a la pregunta realizada antes y después de la MSC “¿Podrías ofrecer algún bien o servicio dentro de la escuela?", que muestra un incremento de un $+0,34$ pts. En este sentido, los alumnos estimulan su capacidad emprendedora al buscar productos y servicios que ellos mismo pudieran ofertar. Por otro lado, al tratarse de una metodología activa, necesariamente se estimula el saber hacer, puesto que son los alumnos quienes hacen su propia moneda.

El cuarto objetivo, "Asumir estrategias de actuación, actividad y reflexión económica", es más complejo de valorar su consecución, debido al escaso horizonte temporal que se desarrollo la innovación docente y la imposibilidad de estar con los alumnos a diario, manteniendo un contacto más directo, e incluso haber desarrollado contenidos que facilitaran la evaluación de su actividad y reflexiones. Aun así, se observan ciertas respuestas que evidencian reflexiones económicas:

- $\quad$ En la pregunta 2 de tipo abierta se encuentran respuestas como: "Facilita a tener más recursos"; en la pregunta 4: "Que no se necesita dinero real como el euro, ni es controlado por el banco de España"; o en la pregunta 6: "Que haya más cosas para ofrecer, no sólo clases, sino también productos alimenticios hechos por nosotros, etc.". Respuestas todas ellas que dejan ver esas reflexiones de las que se habla en el cuarto objetivo.

- También se aprecia como los alumnos llegan a profundizar en que la MSC creada es una herramienta real de intercambio, pues después de haberla utilizado ya no ven la moneda como una herramienta que tiene que ser formalizada y aceptada por las autoridades legales y económicas: "Poco útil, porque no tendría valor fuera de aquí" - "No tendría valor real económico" - "no tiene apoyo de los demás países". $\mathrm{Y}$ sin en cambio, tras el poco tiempo de uso que han tenido los alumnos el mensaje es otro, es de utilidad si hay ofertas variadas.

(cc) EY-NC-ND 2016, Universitat Politècnica de València

Congreso In-Red (2016) 
Sobre el quinto objetivo, "Generar intercambios de bienes y servicios en el aula", decir que no se han alcanzado niveles óptimos de intercambio esperados ( 0,65 por alumno). Como ya se ha comentado anteriormente, dos causas importantes afectaron a esta circunstancia: imposibilidad de estar en el aula durante el desarrollo de la misma coordinando la herramienta y fomentando los intercambios; y, el escaso horizonte temporal en el que se ha esado operando con la moneda ( 3 semanas), hecho que los alumnos han trasladado en varias de sus respuestas.

Respecto al sexto objetivo, "Mejorar las relaciones entre los alumnos", teniendo en cuenta el poco tiempo de desarrollo de la actividad y valorando la respuesta numérica y las preguntas de opinión se considera que su grado de consecución es neutro, si bien, en las respuestas de opinión los alumnos destacan su potencial de mejorar las relaciones entre ellos. Así, se puede señalar como ejemplos:

- En la pregunta tipo Likert $n^{\circ} 15$, “ $i O s$ ha ayudado a conoceros más entre vosotros?", la respuesta es de 2,98 , puntuación muy próxima a 3 que es el neutro de la escala.

- Entre las respuestas obtenidas en las preguntas abiertas $\mathrm{n}^{0} 2 \mathrm{y} \mathrm{n}^{0} 3$, por ejemplo, se pueden encontrar: "Nos sirve para divertirnos y relacionarnos más entre nosotros"; "Porque serviría de ayuda para mejorar las relaciones en clase"; "Ser mejores compañeros"...

Por tanto, como se ha expuesto a lo largo de estas líneas, analizando los resultados, se puede concluir que la mayoría de los objetivos propuestos en esta innovación docente son alcanzados. La utilización de una herramienta de intercambio como puede ser una MSC se muestra como un buen instrumento para desarrollar en el ámbito educativo. Además, se debe señalar que mejorando aquellos aspectos débiles de la puesta en marcha de la innovación docente, como la cuestión de la temporalización y un contacto más directo con los alumnos, los objetivos previsiblemente serían alcanzados con una mayor profundidad, acorde a los resultados obtenidos en las encuestas.

Por otra parte, como complemento de la fase de análisis y valoración de la innovación docente se muestra un análisis DAFO de la misma (ver tabla 3). Así, este análisis DAFO nos sirve como guía para mejorar la innovación docente en aras de elaborar un proyecto más completo, que encuentre aplicaciones en el ámbito educativo y que puedan tener cabida en las aulas tanto de enseñanzas medias como universitarias. 
Tabla 3: Matriz DAFO de la innovación.

\begin{tabular}{|c|c|}
\hline DEBILIDADES & AMENAZAS \\
\hline Líneas de productos limitados & Que la dirección del colegio no lo vea adecuado \\
\hline Escasa cualificación del personal & Disminución de la demanda de bienes y servicios \\
\hline \multirow{2}{*}{ Poco tiempo de los alumnos } & Pérdida de libretas de usuarios \\
\hline & Pérdida de interés por parte de los participantes \\
\hline FORTALEZAS & OPORTUNIDADES \\
\hline Aumenta las interacciones entre los alumnos & Aumentar el ambito de actuación (curso, colegio, barrio...) \\
\hline Promueve la solidaridad interna & Utilizar las TIC's para mejorar la gestión \\
\hline Recursos financieros adecuados & Facilitar procesos de participación de la comunidad educativa \\
\hline Soporte para aprender contenidos de la materia & Fomentar las habilidades de los alumnos \\
\hline \multirow[t]{2}{*}{ Aumenta los recursos de los participantes } & Unirse a otras comunidades de intercambio \\
\hline & Aumentar la variedad de bienes y servicios. \\
\hline
\end{tabular}

Fuente: Elaboración Propia.

\section{Conclusiones}

Este trabajo muestra una innovación docente que consiste en el uso de una Moneda Social y Complementaria el aula (MSC).

Con la aplicación de la misma, durante un mes, en dos grupos-clases diferentes de $1^{\circ}$ bachillerato pertenecientes al mismo instituto situado en la localidad de Sevilla y con un total de 31 alumnos, se ha pretendido realizar una primera experiencia que sirviera para analizar la viabilidad de dicha innovación docente, con el objeto de seguir mejorando la misma para obtener un proyecto más completo y aplicable al ámbito educativo tanto de enseñanzas medias como universitarias, contribuyendo de esta forma a establecer puntos de encuentro entre ambos niveles educativos.

Mediante la implantación en el aula de esta innovación se pretendía alcanzar los siguientes objetivos: 1) Motivar a los alumnos en su aprendizaje, 2) Mejorar el conocimiento de los contenidos relacionados con Monedas y Mercado, 3) Estimular el "saber hacer" y la capacidad emprendedora, 4) Asumir estrategias de actuación, actividad y reflexión económica, 5) Generar intercambios de bienes y servicios en el aula, 6) Mejorar las relaciones entre los alumnos.

Por medio de la metodología, tanto cuantitativa como cualitativa, basada en la obtención de información mediante una serie de preguntas previas y posteriores al uso de la MSC, y utilizando la escala Likert y preguntas abiertas, se ha conseguido analizar el grado de consecución de los objetivos planteados. Los resultados demuestran que la mayoría de los objetivos se han logrado y, por tanto, la aplicación de una MSC en el aula permite mejorar el nivel social (mejor relación entre los alumnos), el nivel académico (como aumentar la cultura financiera y la motivación) y fomentar la iniciativa individual y toma de decisiones.

Si bien, en la aplicación de esta innovación docente no se ha estado exento de ciertas limitaciones, como la imposibilidad de estar en clase durante el desarrollo de la MSC y que el plazo de tiempo que estuvo operativa la MSC fue bastante corto (3 semanas del mes total que duró la innovación docente).

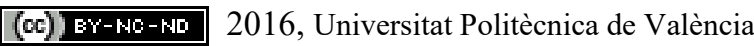

Congreso In-Red (2016) 
Así mismo, como se ha expuesto, este era un primer paso en el trabajo de esta innovación educativa. A continuación se detallan algunos futuros pasos o líneas de trabajo que contribuirán a la mejora y desarrollo de la innovación educativa aquí presentada:

- Implantar la herramienta de intercambio lo antes posible para poder utilizarla durante más tiempo. De esta manera la MSC estará presente durante el desarrollo de los contenidos de la materia y con ello se podrá valorar adecuadamente el objetivo número cuatro "Asumir estrategias de actuación, actividad y reflexión económica".

- Lograr poder tener una experiencia en el aula como "observador participante", durante el desarrollo de la MSC.

- Realizar entrevistas semiestructuradas a varios usuarios de manera individual: estudiantes participantes, no participantes y otros profesores. Así como sesiones de control y evaluación intermedias.

- Potenciar algunos de los factores importantes para una puesta exitosa de la herramienta de intercambio. Como, por ejemplo, la utilización de las TICs para las anotaciones de los intercambios (vease la Web CES) o bases de datos de herramientas de intercambio; consensuar el mecanismo y objetivo del sistema; o, garantizar una suficiente variedad de productos y servicios que cubran necesidades de los alumnos.

- Buscar ideas dentro de "la economía del don", "banco de conocimientos" (ver, por ejemplo, web de YOUCOOP), "banco del tiempo" y de trueque.

- Trabajar en actividades complementarias que favorezcan el desarrollo y la implantación de la MSC. Así, se puede proponer la realización de "mercadillos", espacios físicos y temporales de intercambio y otras actividades lúdicas, recreativas y culturales.

- Intentar implementar la MSC a nivel de curso o mayor rango, ampliando así el número de alumnos y, por tanto, de posibilidades de intercambio, dotando de mayor dinamismo a la MSC.

Como se puede observar las posibilidades son cuantiosas, debiendo definir claramente cuál es el objetivo perseguido para evitar dispersiones que puedan derivar en el fracaso de la innovación docente.

En conclusión, la innovación docente que aquí se presenta, se muestra como un buen medio y una buena herramienta pedagógica para su implantación en las aulas.

\section{Referencias}

Blanc, J. (2009). "Contraintes et choix organisationnels dans les dispositifs de monnaies sociales" en Annals of Public and Cooperative Economics, $n^{\circ}$ 80, Vol. 40, pp.547-577.

(cc)) EY-NC-ND 2016, Universitat Politècnica de València 
Douthwaite, R. (1999). The Ecology of Money, Greenbooks, Totnes (Reino Unido).

Hirota, Y. (2013). Monedas sociales y complementarias (MSC's): Experiencias, su papel en la economía social, estrategias, marketing y políticas públicas. Universitat de València. Martin Gordillo, M. (2006). "Conocer, manejar, valorar, participar: los fines de una educación para la ciudadanía" en Revista Iberoamericana de Educación, ${ }^{\circ}$ 42, pp. 69-84. North, P. (2010). Local Money - How to make it happen in your community, Transition Books, Totnes (Inglaterra).

Primavera, H. (1999). "La moneda social de la Red Global de Trueque en Argentina: ¿barajar y dar de nuevo en el juego social?" en Actas del Seminario Internacional sobre "Globalización de los Mercados Financieros y sus efectos en los países emergentes», organizado por el Instituto Internacional Jacques Maritain, la CEPAL y el Gobierno de Chile, Santiago 29-31 marzo 1999.

Páginas Web:

YOUCOOP. Banco de conocimiento: http://www.youcoop.org/es/about/

CES. Community Exchange System: https://www.community-exchange.org/index_es.asp El Puma. Red de moneda social: http://monedasocialpuma.wordpress.com/tag/monedasocial-puma/ 